\title{
Interaction of a dipole with an interfacial crack in piezoelectric media
}

\author{
Cun-Fa Gao, Pin Tong and Tong-Yi Zhang* \\ Department of Mechanical Engineering, Hong Kong University of Science and Technology \\ Clear Water Bay, Kowloon, Hong Kong, P.R. China
}

\begin{abstract}
We have studied the interaction of an electric dipole with an interface crack between two dissimilar piezoelectric materials. The solutions are derived based on the Stroh formalism. Three crack models studied here are the electrically impermeable, permeable and conducting cracks. The Green functions, the intensity factors of fields and the energy release rate are presented for the three cases. Numerical calculations have also performed to investigate the effect of the dipole's rotation on the energy release rate at the crack tip. It is shown that the energy release rates for the three crack models all reach their maximum values when the direction of the dipole is perpendicular to the crack. Moreover, the energy release rate for an electrically conducting crack is greater than that for an electrically impermeable or permeable crack having the same size under the same loading conditions.
\end{abstract}

Keywords: A. Piezoelectric Ceramics; C. Cracks; Electric dipole; Energy release rate

\footnotetext{
${ }^{*}$ Corresponding author, Tel. +852-2358-7193, Fax +852-2358-1543, E-mail: mezhangt@ust.hk
} 


\section{Introduction}

Piezoelectric materials have found wide applications in electromechanical sensors, transducers and actuators [1]. The most widely used piezoelectric material is ferroelectric. For ferroelectric materials, at temperatures higher than the Curie point, the local positive center of a unit lattice cell coincides with the negative charge center of the cell. When the temperature is lower, spontaneous polarization occurs along crystalline directions, wherein the local positive charge centre of a unit cell deviates from the negative charge centre. The deviation generates an electric dipole as well as internal local strains. The region with uniform polarization is called an electric domain and a ferroelectrtic material has a domain structure, which is usually consisted of electric domains and domain walls. Under external applied electrical and/or mechanical fields, the polarization in each domain may be reoriented and the domain structure may be completely changed, which is generally called domain switching.

Domain switching is the main source of causing nonlinear behaviours of ferroelectric materials including the nonlinear fracture behavior. Based on the domain-level events, Fulton and Gao [2] proposed a two dimensional microstructural model to study the fracture behaviour of an electrically impermeable crack in a ferroelectric ceramic. In their model, the material is divided into many discrete electric domains. An electric dipole and a force couple are used to represent the spontaneous polarization and strains, respectively, in each domain. When the local stress intensity factor is used as the fracture criterion, the numerical simulation results agree well with the experimental observations [3]. 
Green's functions for electric dipole and force couple are the foundations in micromechanics analysis of piezoelectric solids. Wang and Kuang [4] derived the Green functions for a finite crack in piezoelectric material. Gao and Balke [5] solved a similar problem for an electrically permeable crack in a homogeneous piezoelectric medium. They gave the explicit expressions of the Green functions and the electric field within the crack induced by an electric dipole and a force couple.

In the present work, we study the interaction of an electric dipole with an interface crack between two dissimilar piezoelectric materials. Using the Stroh formalism (Section 2), we first derive Green's functions, the field intensity factors and the energy release rate for an electric dipole located near an impermeable interface crack (Section 3). In section 4, we extend these results to the case of conducting interface crack by introducing the mixed-form Stroh formalism. The corresponding solutions for a permeable interface crack are given in Section 5. Section 6 presents the numerical results of the energy release rate $G$ for the three crack models to illustrate the influence of the dipole rotation on the piezoelectric fracture. Finally, Section 7 concludes the present work.

\section{Stroh formalism}

In this section we briefly outline the standard Stroh formalism for later use. The Stroh formalism has been widely used in the two-dimensional theoretical analysis of anisotropic elastic materials [6] and piezoelectric media [1,7]. Note that matrix and vector notations we use in this article are more consistent with those used in Reference [7], but may differ from the notations used in the Reference [1]. 
In a rectangular coordinate system, $x_{i}(i=1,2,3)$, the complete set of basic equations for a linear piezoelectric solid without any body forces and free charges is given by the equilibrium equation, (1), and the kinematic equation, (2), and the constitutive equation, (3), as

$$
\begin{aligned}
& \sigma_{i j, j}=0, \quad D_{i, i}=0, \\
& \varepsilon_{i j}=\frac{1}{2}\left(u_{i, j}+u_{j, i}\right), \quad E_{i}=-\psi_{, i}, \\
& \sigma_{i j}=C_{i j k l} \varepsilon_{k l}-e_{k j} E_{k}, \\
& D_{k}=e_{k j} \varepsilon_{i j}+\kappa_{k l} E_{l},
\end{aligned}
$$

where $\sigma_{i j}, \varepsilon_{i j}, D_{i}$ and $E_{i}$ denote stress tensor, strain tensor, electric displacement vector and electric field vector, respectively; $u_{i}$ and $\psi$ are the elastic displacements and electric potential, respectively; and $C_{i j k l}, e_{i j k}$ and $\kappa_{i j}$ stand for the elastic constants, the piezoelectric constants and the dielectric constants, respectively.

For generalized two-dimensional deformations in which the generalized displacement vector $\mathbf{u}=\left(\begin{array}{llll}u_{1} & u_{2} & u_{3} & \psi\end{array}\right)^{T}$ depends on $x_{1}$ and $x_{2}$ only, the general solution takes the form:

$$
\begin{aligned}
& \mathbf{u}=\mathbf{A f}(z)+\overline{\mathbf{A}} \overline{\mathbf{f}(z)}, \\
& \phi=\mathbf{B f}(z)+\overline{\mathbf{B}} \overline{\mathbf{f}(z)},
\end{aligned}
$$

where $\mathbf{A}=\left(\begin{array}{llll}\mathbf{a}_{1} & \mathbf{a}_{2} & \mathbf{a}_{3} & \mathbf{a}_{4}\end{array}\right)$ and $\mathbf{B}=\left(\begin{array}{lllll}\mathbf{b}_{1} & \mathbf{b}_{2} & \mathbf{b}_{3} & \mathbf{b}_{4}\end{array}\right)$ with $\mathbf{a}_{\alpha}$ and $\mathbf{b}_{\alpha}$ for $\alpha=1,2,3,4$ being both four dimensional eigen-vectors, $\mathbf{f}(\mathrm{z})=\left(\begin{array}{llll}f_{1}\left(z_{1}\right) & f_{2}\left(z_{2}\right) & f_{3}\left(z_{3}\right) & f_{4}\left(z_{4}\right)\end{array}\right)^{T}$ is an analytic function vector, $z_{\alpha}=x_{1}+p_{\alpha} x_{2}$, and $p_{\alpha}$ is a complex eigen-root with a positive imaginary part, and $\phi$ is the generalized stress function vector such that 


$$
\begin{aligned}
& \Sigma_{2}=\left(\begin{array}{llll}
\sigma_{21} & \sigma_{22} & \sigma_{23} & D_{2}
\end{array}\right)^{T}=\phi_{, 1}, \\
& \Sigma_{1}=\left(\begin{array}{llll}
\sigma_{11} & \sigma_{12} & \sigma_{13} & D_{1}
\end{array}\right)^{T}=-\phi_{, 2} .
\end{aligned}
$$

It is convenient to calculate $p_{\alpha}$ by solving the following standard eigen-equation:

$$
\left(\begin{array}{ll}
\mathbf{N}_{1} & \mathbf{N}_{2} \\
\mathbf{N}_{3} & \mathbf{N}_{1}^{T}
\end{array}\right)\left(\begin{array}{l}
\mathbf{a} \\
\mathbf{b}
\end{array}\right)=p\left(\begin{array}{l}
\mathbf{a} \\
\mathbf{b}
\end{array}\right)
$$

where $\quad \mathbf{N}_{1}=-\mathbf{T}^{-1} \mathbf{R}^{T}, \quad \mathbf{N}_{2}=\mathbf{T}^{-1}=\mathbf{N}_{2}^{T}, \quad \mathbf{N}_{3}=\mathbf{R} \mathbf{T}^{-1} \mathbf{R}^{T}-\mathbf{Q}=\mathbf{N}_{3}^{T}, \quad$ and $\quad \mathbf{Q}=\left(\begin{array}{cc}C_{i 1 k 1} & e_{11 i} \\ e_{11 i}^{T} & -\kappa_{11}\end{array}\right)$ $\mathbf{R}=\left(\begin{array}{cc}C_{i 1 k 2} & e_{21 i} \\ e_{12 i}^{T} & -\kappa_{12}\end{array}\right), \mathbf{T}=\left(\begin{array}{cc}C_{i 2 k 2} & e_{22 i} \\ e_{22 i}^{T} & -\kappa_{22}\end{array}\right), i, k=1,2,3$. The $\mathbf{A}$ and $\mathbf{B}$ matrices have the following relationship:

$$
\left(\begin{array}{ll}
\mathbf{B}^{T} & \mathbf{A}^{T} \\
\overline{\mathbf{B}}^{T} & \overline{\mathbf{A}}^{T}
\end{array}\right)\left(\begin{array}{ll}
\mathbf{A} & \overline{\mathbf{A}} \\
\mathbf{B} & \overline{\mathbf{B}}
\end{array}\right)=\left(\begin{array}{ll}
\mathbf{I} & 0 \\
0 & \mathbf{I}
\end{array}\right)
$$

where $\mathbf{I}$ is a $4 \times 4$ unit matrix. In addition, two matrices, $\mathbf{Y}$ and $\mathbf{H}$, are often used in the following analysis, which are defined by

$$
\mathbf{Y}=i \mathbf{A B}^{-1}, \mathbf{H}=2 \operatorname{Re}[\mathbf{Y}]
$$

Matrix $\mathbf{Y}$ is a Hermitian matrix and can be partitioned into

$$
\mathbf{Y}=\left(\begin{array}{cc}
\mathbf{Y}_{e} & \mathbf{Y}_{31} \\
\mathbf{Y}_{13} & Y_{44}
\end{array}\right)
$$

where the upper left block, $\mathbf{Y}_{e}$, is a $3 \times 3$ matrix, and $Y_{44}$ is a real element. For a stable material, $\mathbf{Y}_{e}$ is positive definite and $Y_{44}<0$, which leads to $H_{44}<0$. For use convenience, we take partial differentiation of (4) with respect to $x_{1}$ and have the forms of

$$
\phi_{, 1}=\mathbf{B F}(z)+\overline{\mathbf{B}} \overline{\mathbf{F}(z)}
$$




$$
\mathbf{u}_{, 1}=\frac{1}{i}[\mathbf{Y B F}(z)-\overline{\mathbf{Y B}} \overline{\mathbf{F}}(z)]
$$

where $\mathbf{F}(z)=d \mathbf{f}(z) / d z$.

\section{Solution for an impermeable interface crack}

\subsection{Statement of the problem}

Consider a dipole, $\mathbf{p}_{0}$, with the dipole moment magnitude of $p_{0}$ located at $z_{0}=x_{10}+i x_{20}$ in the upper half-plane, $s_{1}$. On the interface between $s_{1}$ and the lower half-plane $s_{2}$, there is an impermeable crack denoted by $L_{c}$, as shown in Fig.1. The traction-free boundary conditions along the crack faces take the mathematic form of [8]:

$$
\begin{gathered}
\sigma_{2 j}^{+}\left(x_{1}\right)=\sigma_{2 j}^{-}\left(x_{1}\right)=0,(j=1,2,3), \text { on } \mathrm{L}_{\mathrm{c}}, \\
D_{2}^{+}\left(x_{1}\right)=D_{2}^{-}\left(x_{1}\right)=0, \text { on } \mathrm{L}_{\mathrm{c}} .
\end{gathered}
$$

We decompose the problem into two subproblems: subproblem A is an uncracked bimaterail system with the dipole $\mathbf{p}_{0}$ and subproblem B is a cracked bimaterail system with loads on the crack faces. According to supposition principle, the complex vector, $\mathbf{F}(z)$, introduced in (9), can be expressed as

$$
\mathbf{F}(z)=\mathbf{F}_{a}(z)+\mathbf{F}_{b}(z),
$$

where $\mathbf{F}_{a}(z)$ and $\mathbf{F}_{b}(z)$ are the complex solution vectors for subproblems A and B, respectively, in which $\mathbf{F}_{b}(\infty)=\mathbf{0}$.

For sub-problem A, shown in Fig.2, the continuous conditions along the whole the $x_{1}$ axis require

$$
\phi_{a, 1}^{+}\left(x_{1}\right)=\phi_{a, 1}^{-}\left(x_{1}\right), \quad \mathbf{u}_{a, 1}^{+}\left(x_{1}\right)=\mathbf{u}_{a, 1}^{-}\left(x_{1}\right), \quad-\infty<x_{1}<\infty,
$$

where $\phi_{a, 1}$ and $\mathbf{u}_{a, 1}$ denote the generalized stress and strain vectors generated by the dipole $\mathbf{p}_{0}$ such that 


$$
\begin{aligned}
& \phi_{a, 1}\left(x_{1}\right)=\mathbf{B} \mathbf{F}_{a}\left(x_{1}\right)+\overline{\mathbf{B}} \overline{\mathbf{F}_{a}\left(x_{1}\right)}, \\
& \mathbf{u}_{a, 1}\left(x_{1}\right)=\mathbf{A} \mathbf{F}_{a}\left(x_{1}\right)+\overline{\mathbf{A}} \overline{\mathbf{F}_{a}\left(x_{1}\right)} .
\end{aligned}
$$

Substituting (12) into (9) leads to

$$
\begin{aligned}
& \phi_{, 1}=\phi_{a, 1}+\mathbf{B} \mathbf{F}_{b}(z)+\overline{\mathbf{B}} \overline{\mathbf{F}_{b}(z)} \\
& \mathbf{u}_{, 1}=\mathbf{u}_{a, 1}+\frac{1}{i}\left[\mathbf{Y B F}_{b}(z)-\overline{\mathbf{Y}} \overline{\mathbf{B}} \overline{\mathbf{F}_{b}(z)}\right] .
\end{aligned}
$$

Since the complex vector for sub-problem A can be easily obtain, see Appendix A, our task below is to find $\mathbf{F}_{b}(z)$ by solving sub-problem B.

\subsection{Complex potentials}

On the entire $x_{1}$ axis, the continuity condition of the generalized stress is

$$
\phi_{, 1}^{+}\left(x_{1}\right)=\phi_{, 1}^{-}\left(x_{1}\right), \quad-\infty<x_{1}<\infty .
$$

Substituting (16) into (18) and then using (13) leads to

$$
\mathbf{B}_{\mathbf{1}} \mathbf{F}_{\mathbf{1} b}^{+}\left(x_{1}\right)+\overline{\mathbf{B}}_{\mathbf{1}} \overline{\mathbf{F}}_{\mathbf{1} b}^{-}\left(x_{1}\right)=\mathbf{B}_{2} \mathbf{F}_{2 b}^{-}\left(x_{1}\right)+\overline{\mathbf{B}}_{2} \overline{\mathbf{F}}_{2 b}^{+}\left(x_{1}\right), \quad-\infty<x_{1}<\infty .
$$

Following Muskhelishvili's theory (1975), we have from (19) that

$$
\begin{array}{ll}
\mathbf{B}_{1} \mathbf{F}_{1 b}(z)-\overline{\mathbf{B}}_{2} \overline{\mathbf{F}}_{2 b}(z)=\mathbf{0}, & z \in s_{1}, \\
\mathbf{B}_{2} \mathbf{F}_{2 b}(z)-\overline{\mathbf{B}}_{1} \overline{\mathbf{F}}_{1 b}(z)=\mathbf{0}, & z \in s_{2} .
\end{array}
$$

where $\overline{\mathbf{F}}_{b}(z)=\overline{\mathbf{F}_{b}(\bar{z})}$.

Using (20) and (21), we obtain from (16) and (17) that

$$
\begin{aligned}
& \phi_{, 1}^{+}\left(x_{1}\right)=\phi_{a, 1}^{+}\left(x_{1}\right)+\mathbf{B}_{1} \mathbf{F}_{1 b}^{+}\left(x_{1}\right)+\mathbf{B}_{2} \mathbf{F}_{2 b}^{-}\left(x_{1}\right), \\
& \mathbf{u}_{, 1}^{+}\left(x_{1}\right)=\mathbf{u}_{a, 1}^{+}\left(x_{1}\right)+\frac{1}{i}\left[\mathbf{Y}_{1} \mathbf{B}_{1} \mathbf{F}_{1 b}^{+}\left(x_{1}\right)-\overline{\mathbf{Y}}_{1} \mathbf{B}_{2} \mathbf{F}_{2 b}^{-}\left(x_{1}\right)\right], \\
& \mathbf{u}_{, 1}^{-}\left(x_{1}\right)=\mathbf{u}_{a, 1}^{-}\left(x_{1}\right)+\frac{1}{i}\left[\mathbf{Y}_{2} \mathbf{B}_{2} \mathbf{F}_{2 b}^{-}\left(x_{1}\right)-\overline{\mathbf{Y}}_{2} \mathbf{B}_{1} \mathbf{F}_{1 b}^{+}\left(x_{1}\right)\right] .
\end{aligned}
$$

Let us introduce a jump function:

$$
i \Delta \mathbf{u}_{, 1}\left(x_{1}\right)=i\left[\mathbf{u}_{, 1}^{+}\left(x_{1}\right)-\mathbf{u}_{, 1}^{-}\left(x_{1}\right)\right] .
$$


Then, inserting (23) and (24) into (25) and using (13) $)_{2}$ result in

$$
i \Delta \mathbf{u}_{, 1}\left(x_{1}\right)=\mathbf{K}^{+}\left(x_{1}\right)-\mathbf{K}^{-}\left(x_{1}\right),
$$

where

$$
\begin{gathered}
\mathbf{K}(z)=\left\{\begin{array}{l}
\mathbf{H B}_{1} \mathbf{F}_{1 b}(z), z \in s_{1} \\
\overline{\mathbf{H}}_{2} \mathbf{F}_{2 b}(z), z \in s_{2}
\end{array},\right. \\
\mathbf{H}=\mathbf{Y}_{1}+\overline{\mathbf{Y}}_{2} .
\end{gathered}
$$

On the other hand, (22) can be rewritten as

$$
\phi_{, 1}^{+}\left(x_{1}\right)=\phi_{p, 1}^{+}\left(x_{1}\right)+\mathbf{h} \mathbf{K}^{+}\left(x_{1}\right)+\overline{\mathbf{h}} \mathbf{K}^{-}\left(x_{1}\right),
$$

where $\mathbf{h}=\mathbf{H}^{-1}$. Equation (29) can be reduced to

$$
\mathbf{h}^{-1} \phi_{, 1}^{+}\left(x_{1}\right)=\mathbf{h}^{-1} \boldsymbol{\phi}_{a, 1}^{+}\left(x_{1}\right)+\mathbf{K}_{3}^{+}\left(x_{1}\right)+\mathbf{h}^{-1} \overline{\mathbf{h}} \mathbf{K}_{3}^{-}\left(x_{1}\right) .
$$

Letting $\mathbf{Q}$ be the eigenvector matrix of $\mathbf{h}^{-1} \overline{\mathbf{h}}$, one has

$$
\mathbf{Q}^{-1} \mathbf{h}^{-1} \overline{\mathbf{h}} \mathbf{Q}=\left\langle\left\langle e^{2 \pi \varepsilon_{\alpha}}\right\rangle\right\rangle,
$$

where the angular bracket $\langle\langle\rangle\rangle$ indicates the diagonal matrix in which each component varies according to the Greek index $\alpha$, and $\varepsilon_{\alpha}$ are determined by

$$
\left\|\overline{\mathbf{h}}-e^{2 \pi \varepsilon_{\alpha}} \mathbf{h}^{-1}\right\|=0
$$

Multiplying two sides of (30) by $\mathbf{Q}^{-1}$ and then using (31), we find

$$
\mathbf{M} \phi_{, 1}^{+}\left(x_{1}\right)=\mathbf{R}_{0}\left(x_{1}\right)+\mathbf{R}^{+}\left(x_{1}\right)+\left\langle\left\langle e^{2 \pi \varepsilon_{\alpha}}\right\rangle\right\rangle \mathbf{R}^{-}\left(x_{1}\right)
$$

where

$$
\mathbf{M}=[\mathbf{h} \mathbf{Q}]^{-1}, \quad \mathbf{R}_{0}\left(x_{1}\right)=\mathbf{M} \phi_{a, 1}\left(x_{1}\right), \mathbf{R}(z)=\mathbf{Q}^{-1} \mathbf{K}(z)
$$

On the crack faces, the condition of free traction means

$$
\phi_{, 1}^{+}\left(x_{1}\right)=0, \quad x_{1} \in L_{c} .
$$

Substituting (34) into (32) yields

$$
\mathbf{R}^{+}\left(x_{1}\right)+\left\langle\left\langle e^{2 \pi \varepsilon_{\alpha}}\right\rangle\right\rangle \mathbf{R}^{-}\left(x_{1}\right)=-\mathbf{R}_{0}\left(x_{1}\right), \quad x_{1} \in L_{c} .
$$


Expanding (35) results in

$$
R_{\alpha}^{+}\left(x_{1}\right)+e^{2 \pi \varepsilon_{\alpha}} R_{\alpha}^{-}\left(x_{1}\right)=-R_{\alpha 0}\left(x_{1}\right), \quad \alpha=1-4 .
$$

The general solution of (36) is [9]

$$
R_{\alpha}(z)=\frac{I_{\alpha}(z)}{X_{\alpha}(z)}+\frac{c_{\alpha 0}}{X_{\alpha}(z)}, \quad \alpha=1-4,
$$

where $c_{\alpha 0}$ is a constant to be determined and

$$
\begin{aligned}
X_{\alpha}(z) & =\sqrt{z^{2}-a^{2}}\left(\frac{z+a}{z-a}\right)^{i \varepsilon_{\alpha}}, \\
I_{\alpha}(z) & =\frac{1}{2 \pi i} \int_{L_{c}} \frac{-X_{\alpha}^{+}\left(x_{1}\right) R_{\alpha 0}\left(x_{1}\right) d x_{1}}{x_{1}-z} .
\end{aligned}
$$

In order to find $c_{\alpha 0}$, one has to use the single-valued condition of generalized displacements such as

$$
\int_{L_{c}} \Delta \mathbf{u}_{, 1}\left(x_{1}\right) d x_{1}=\mathbf{0} .
$$

Substituting (26) into (40) leads to

$$
\int_{L_{c}}\left[\mathbf{K}^{+}\left(x_{1}\right)-\mathbf{K}^{-}\left(x_{1}\right)\right] d x_{1}=\mathbf{0} .
$$

Multiplying $\mathbf{Q}^{-1}$ in both sides of (41) gives

$$
\int_{L_{c}}\left[\mathbf{R}^{+}\left(x_{1}\right)-\mathbf{R}^{-}\left(x_{1}\right)\right] d x_{1}=0,
$$

that is

$$
\int_{L_{c}}\left[R_{\alpha}^{+}\left(x_{1}\right)-R_{\alpha}^{-}\left(x_{1}\right)\right] d x_{1}=0, \quad \alpha=1-4 .
$$

To determine the complete form of $R_{\alpha}(z), R_{\alpha 0}\left(x_{1}\right)$ in (39) has to be given. According to (33) $R_{\alpha 0}\left(x_{1}\right)$ can be expressed as

$$
R_{\alpha 0}\left(x_{1}\right)=\sum_{j=1}^{4} M_{\alpha j} \phi_{a, 1 j}\left(x_{1}\right),
$$

where $\phi_{a, 1 j}\left(x_{1}\right)$ can be obtained from (A6) in Appendix A, that is

$$
\phi_{a, 1 j}\left(x_{1}\right)=p_{0} \sum_{k=1}^{4}\left[N_{j k} \varphi_{k} q_{k}\left(x_{1}-z_{k 0}\right)^{-2}+\overline{N_{j k} \varphi_{k} q_{k}}\left(x_{1}-\bar{z}_{k 0}\right)^{-2}\right], \quad j=1-4 .
$$

Substituting (45) into (44), we obtain 


$$
R_{\alpha 0}\left(x_{1}\right)=p_{0} \sum_{j=1}^{4} \sum_{k=1}^{4} M_{\alpha j}\left[N_{j k} \varphi_{k} q_{k}\left(x_{1}-z_{k 0}\right)^{-2}+\overline{N_{j k} \varphi_{k} q_{k}}\left(x_{1}-\bar{z}_{k 0}\right)^{-2}\right] .
$$

Substituting (46) into (39) and then using Muskhelishvili's theory [9] and (43), we can determine $R_{\alpha}(z)$ and $c_{\alpha 0}$. The results are $c_{\alpha 0}=0$ and

$$
\begin{aligned}
R_{\alpha}(z)= & -\frac{1}{1+e^{2 \pi \varepsilon_{\alpha}}} R_{\alpha 0}(z)+ \\
& +\frac{p_{0}}{X_{\alpha}(z)\left(1+e^{2 \pi \varepsilon_{\alpha}}\right)} \sum_{j=1}^{4} \sum_{k=1}^{4} M_{\alpha j}\left[N_{j k} \varphi_{k} q_{k} \Omega_{\alpha}\left(z, z_{k 0}\right)+\overline{N_{j k} \varphi_{k} q_{k}} \Omega_{\alpha}\left(z, \bar{z}_{k 0}\right)\right],
\end{aligned}
$$

where

$$
\Omega_{\alpha}\left(z, z_{k 0}\right)=\frac{X_{\alpha}\left(z_{k 0}\right)}{\left(z-z_{k 0}\right)^{2}}+\frac{X_{\alpha}^{\prime}\left(z_{k 0}\right)}{z-z_{k 0}} .
$$

We then have $\mathbf{K}(z)=\mathbf{Q R}(z)$. Substituting $\mathbf{K}(z)$ into (27), one can finally determine the complex potentials $\mathbf{F}_{k b}(z)(k=1,2)$. All field variables in $s_{1}$ and $s_{2}$ are now determined.

\subsection{Intensity factors of fields}

At the right crack-tip, the intensity factor vector is defined as $[10,11]$

$$
\mathbf{k}=\left[k_{I I}, k_{I,} k_{I I I}, k_{D}\right]^{T}=\lim _{r \rightarrow 0} \sqrt{2 \pi r} \mathbf{M}^{-1}\left\langle\left\langle r^{-i \varepsilon_{\alpha}}\right\rangle\right\rangle \mathbf{M}_{, 1}^{+}\left(x_{1}\right), \quad x_{1} \in L_{b},
$$

where $L_{b}$ stands for the uncracked part on the interface, $r$ is the distance from the crack-tip along the interface, and $x_{1}=a+r$.

Noting (26), one finds that, ahead of the crack tip, $\mathbf{K}^{+}\left(x_{1}\right)=\mathbf{K}^{-}\left(x_{1}\right)$, since $\Delta \mathbf{u}_{, 1}\left(x_{1}\right)=\mathbf{0}$. From $(33)_{3}$, this means that

$$
\mathbf{R}^{+}\left(x_{1}\right)=\mathbf{R}^{-}\left(x_{1}\right), \quad x_{1} \in L_{b}
$$

Using (50), we can write (32) as

$$
\mathbf{M} \phi_{, 1}^{+}\left(x_{1}\right)=\mathbf{R}_{0}\left(x_{1}\right)+\left\langle\left\langle 1+e^{2 \pi \varepsilon_{\alpha}}\right\rangle\right\rangle \mathbf{R}\left(x_{1}\right), \quad x_{1} \in L_{b} .
$$

Inserting (51) into (49) results in the general expression of stress intensity factors as

$$
\mathbf{k}=\lim _{r \rightarrow 0} \sqrt{2 \pi r} \mathbf{M}^{-1}\left\langle\left\langle\left(1+e^{2 \pi \varepsilon_{\alpha}}\right) r^{-i \varepsilon_{\alpha}}\right\rangle\right\rangle \mathbf{R}\left(x_{1}\right), \quad x_{1} \in L_{b} .
$$


For the sake of explicitness, we rewrite (52) as

$$
\mathbf{M k}=\lim _{r \rightarrow 0} \sqrt{2 \pi r}\left\langle\left\langle\left(1+e^{2 \pi \varepsilon_{\alpha}}\right) r^{-i \varepsilon_{\alpha}}\right\rangle\right\rangle \mathbf{R}\left(x_{1}\right),
$$

and then expand (53) into

$$
\sum_{\beta=1}^{4} M_{\alpha \beta} k_{\beta}=\lim _{r \rightarrow 0} \sqrt{2 \pi r}\left(1+e^{2 \pi \varepsilon_{\alpha}}\right) r^{-i \varepsilon_{\alpha}} R_{\alpha}\left(x_{1}\right), \quad \alpha=1-4 .
$$

Inserting (47) into (54), we have

$$
\sum_{\beta=1}^{4} M_{\alpha \beta} k_{\beta}=p_{0} \sqrt{\frac{\pi}{a}}(2 a)^{-i \varepsilon_{\alpha}} \sum_{j=1}^{4} \sum_{k=1}^{4} M_{\alpha j}\left[N_{j k} \varphi_{k} q_{k} \Omega_{\alpha}\left(a, z_{k 0}\right)+\overline{N_{j k} \varphi_{k} q_{k}} \Omega_{\alpha}\left(a, \bar{z}_{k 0}\right)\right],
$$

or

$$
(2 a)^{i \varepsilon_{\alpha}} \sum_{\beta=1}^{4} M_{\alpha \beta} k_{\beta}=p_{0}\left(c_{\alpha 1} \cos \varphi+c_{\alpha 2} \sin \varphi\right), \alpha=1-4,
$$

where $c_{\alpha 1}$ and $c_{\alpha 2}$ are two coefficients related to only the material constants and the location of the dipole such that

$$
\begin{aligned}
c_{\alpha 1} & =\sqrt{\frac{\pi}{a}} \sum_{j=1}^{4} \sum_{k=1}^{4} M_{\alpha j}\left[N_{j k} q_{k} \Omega_{\alpha}\left(a, z_{k 0}\right)+\overline{N_{j k} q_{k}} \Omega_{\alpha}\left(a, \bar{z}_{k 0}\right)\right], \\
c_{\alpha 2} & =\sqrt{\frac{\pi}{a}} \sum_{j=1}^{4} \sum_{k=1}^{4} M_{\alpha j}\left[N_{j k} p_{k} q_{k} \Omega_{\alpha}\left(a, z_{k 0}\right)+\overline{N_{j k} p_{k} q_{k} \Omega_{\alpha}}\left(a, \bar{z}_{k 0}\right)\right] .
\end{aligned}
$$

Equation (55) can be rewritten in the vector form as

$$
\mathbf{M}\left\langle\left\langle(2 a)^{i \varepsilon_{\alpha}}\right\rangle\right\rangle \mathbf{k}=p_{0}\left(\mathbf{c}_{\alpha 1} \cos \varphi+\mathbf{c}_{\alpha 2} \sin \varphi\right) .
$$

The coefficients $c_{\alpha 1}$ and $c_{\alpha 2}$ are constants, whose values depend the location of the dipole. Thus, using (58), one can study the influence of $p_{0}$ and $\varphi$ on the field singularities.

\subsection{Energy release rate}

For two-dimensional problems, if the crack grows along the crack plane, the energy release rate $\mathrm{G}$ may be calculated from the generalized $J$ integral $[12,1]$

$$
G=J=\int_{\Gamma}\left(W n_{1}-t_{J} u_{J, 1}\right) d \Gamma,
$$


where $W\left(=\frac{1}{2} \Sigma_{i J} u_{J, i}\right)$ is the electric enthalpy density, $n_{i}$ is the unit outward normal vector, $t_{J}$ is the surface traction and the surface electric displacement, given by $t_{J}=n_{i} \Sigma_{i J}$, and $\Gamma$ is a contour enclosing the right crack tip, starting from a point on the lower crack surface and terminating at a point on the upper crack surface. It is well known that the generalized $J$-integral is pathindependent.

The generalized $J$-integral in (59) can be reduced to $[13,14]$

$$
G=J=\operatorname{Re}\left[\sum_{j=1}^{4} \int_{\Gamma}\left\{f_{j}^{\prime}\left(z_{j}\right)\right\}^{2} d z_{j}\right],
$$

Equation (60) can be rewritten as

$$
\left.G=J=\operatorname{Re} \mid \int_{\Gamma} \mathbf{F}_{b}^{T}(z) \mathbf{F}_{b}(z) d z\right\rfloor
$$

After $\mathbf{F}_{b}(z)$ is determined from (27), one can obtain the energy release rate by completing the integral in (61). Some numerical results will be presented in Section 6.

\section{Solution for a conducting interface crack}

For a conducting interface crack, the boundary conditions can be expressed as

$$
\sigma_{2 j}^{+}\left(x_{1}\right)=\sigma_{2 j}^{-}\left(x_{1}\right)=0 \quad(j=1,2,3), E_{1}^{+}\left(x_{1}\right)=E_{1}^{-}\left(x_{1}\right)=0 \text {, on } \mathrm{L}_{\mathrm{c}},
$$

Define two vectors as

$$
\hat{\mathbf{u}}=\left[u_{1}, u_{2}, u_{3}, \phi_{4}\right]^{T}, \hat{\phi}=\left[\phi_{1}, \phi_{2}, \phi_{3}, \psi\right]^{T},
$$

where

$$
\hat{\mathbf{u}}=\mathbf{I}_{u} \mathbf{u}+\mathbf{I}_{t} \phi, \hat{\phi}=\mathbf{I}_{t} \mathbf{u}+\mathbf{I}_{u} \phi
$$

with

$$
\mathbf{I}_{u}=\langle\langle 1,1,1,0\rangle\rangle, \mathbf{I}_{\phi}=\langle\langle 0,0,0,1\rangle\rangle .
$$

Then, substituting (4) into (63) leads to

$$
\hat{\mathbf{u}}=\hat{\mathbf{A}} \mathbf{f}\left(z_{\alpha}\right)+\overline{\hat{\mathbf{A}}} \overline{\mathbf{f}\left(z_{\alpha}\right)}, \hat{\phi}=\hat{\mathbf{B}} \mathbf{f}\left(z_{\alpha}\right)+\overline{\hat{\mathbf{B}}} \overline{\mathbf{f}\left(z_{\alpha}\right)},
$$


where

$$
\hat{\mathbf{A}}=\mathbf{I}_{u} \mathbf{A}+\mathbf{I}_{\phi} \mathbf{B}, \hat{\mathbf{B}}=\mathbf{I}_{\phi} \mathbf{A}+\mathbf{I}_{u} \mathbf{B} .
$$

Equation (64) may be called the Stroh formalism for the mixed boundary value problem. The nature of $\hat{\mathbf{A}}$ and $\hat{\mathbf{B}}$ was studied by Ting [6] and Homulka and Keer [15] for the case in purely anisotropic media, and also by Zhang et al. [1] for piezoelectric materials. It was found that $\hat{\mathbf{A}}$ and $\hat{\mathbf{B}}$ have similar nature to that of $\mathbf{A}$ and $\mathbf{B}$.

If one starts from (64) and follows the similar procedure for impermeable cracks, all equations from (12)-(61) are still valid, as long as one makes the following replacements

$$
(A, B, \mathbf{u}, \phi, \mathbf{Y},) \rightarrow(\hat{A}, \hat{B}, \hat{\mathbf{u}}, \hat{\phi}, \hat{\mathbf{Y}}), \quad \mathbf{q} \rightarrow \frac{1}{2 \pi i} \hat{\mathbf{B}}_{1}^{T} \mathbf{i}_{4}, \quad \mathbf{k} \rightarrow\left[k_{I I}, k_{I}, k_{I I I}-k_{E_{1}}\right]^{T} .
$$

Thus, using the results in Section 3, one can directly obtain the corresponding solution for a conducting interface crack by the replacement (65).

\section{Solution for a permeable interface crack}

Different from the above two crack models where one of electric variables, i.e. $D_{2}^{0}$ or $E_{1}^{0}$, on the crack faces is given, the problem of a permeable crack is more difficult to solve, since all electric variables on the faces of a permeable crack have to be determined as part of solution. In this section, we will introduce some mathematical manipulation to reduce the problem of the permeable interface crack to an equivalent interface crack problem in elastic anisotropic media, and then give its closedform solution both in the media and within the crack.

In this case, the boundary conditions can be written as [16]

$$
\sigma_{2 j}^{+}\left(x_{1}\right)=\sigma_{2 j}^{-}\left(x_{1}\right)=0(j=1,2,3), \quad \text { on } \mathrm{L}_{\mathrm{c}},
$$




$$
D_{2}^{+}\left(x_{1}\right)=D_{2}^{-}\left(x_{1}\right), E_{1}^{+}\left(x_{1}\right)=E_{1}^{-}\left(x_{1}\right), \quad \text { on } \mathrm{L}_{\mathrm{c}} \text {. }
$$

Based on (66) and (67), equations (12)-(29) are still valid, but considering (67) $)_{2}$, one has from (26) that

$$
K_{4}^{+}\left(x_{1}\right)-K_{4}^{-}\left(x_{1}\right)=0,-\infty<x_{1}<\infty
$$

The solution of $(68)$ is

$$
K_{4}(z)=0 .
$$

Equation (69) shows that the fourth component of $\mathbf{K}(z)$ becomes known. Our task below is to find the other three components of $\mathbf{K}(z)$.

To do this, we partition $\mathbf{H}^{-1}$ into four sub-matrices as

$$
\mathbf{h}=\mathbf{H}^{-1}=\left[\begin{array}{ll}
\mathbf{h}_{3 \times 3} & \mathbf{h}_{3 \times 1} \\
\mathbf{h}_{1 \times 3} & \mathbf{h}_{44}
\end{array}\right],
$$

in which $\mathbf{h}_{3 \times 3}$ is a $3 \times 3$ submatrix, $\mathbf{h}_{44}$ is a real element, and $\mathbf{h}_{3 \times 1}$ and $\mathbf{h}_{1 \times 3}$ are $3 \times 1$ and $1 \times 3$ row and column vectors, respectively.

From the first three rows and the final row of (29), respectively, using (69) and (70), we have

$$
\begin{aligned}
& \boldsymbol{\sigma}_{2}\left(x_{1}\right)=\boldsymbol{\sigma}_{2}^{a}\left(x_{1}\right)+\mathbf{h}_{3 \times 3} \mathbf{K}_{3}^{+}\left(x_{1}\right)+\overline{\mathbf{h}}_{3 \times 3} \mathbf{K}_{3}^{-}\left(x_{1}\right), \\
& D_{2}\left(x_{1}\right)=D_{2}^{a}\left(x_{1}\right)+\mathbf{h}_{1 \times 3} \mathbf{K}_{3}^{+}\left(x_{1}\right)+\overline{\mathbf{h}}_{1 \times 3} \mathbf{K}_{3}^{-}\left(x_{1}\right),
\end{aligned}
$$

where

$$
\mathbf{K}_{3}=\left[K_{1}, K_{2}, K_{3}\right]^{T}, \quad \boldsymbol{\sigma}_{2}\left(x_{1}\right)=\left[\sigma_{21}\left(x_{1}\right), \sigma_{22}\left(x_{1}\right), \sigma_{23}\left(x_{1}\right)\right]^{T},
$$

and $\boldsymbol{\sigma}_{2}^{a}\left(x_{1}\right)$ and $D_{2}^{a}\left(x_{1}\right)$ are the stress vector and the electric displacement generated by subproblem A.

Equation (71) can then be reduced to

$$
\mathbf{h}_{3 \times 3}^{-1} \boldsymbol{\sigma}_{2}\left(x_{1}\right)=\mathbf{h}_{3 \times 3}^{-1} \boldsymbol{\sigma}_{2}^{p}\left(x_{1}\right)+\mathbf{K}_{3}^{+}\left(x_{1}\right)+\mathbf{h}_{3 \times 3}^{-1} \overline{\mathbf{h}}_{3 \times 3} \mathbf{K}_{3}^{-}\left(x_{1}\right) .
$$

On the crack faces, the traction free condition means

$$
\boldsymbol{\sigma}_{2}\left(x_{1}\right)=0, \quad x_{1} \in L_{c} .
$$

Substituting (74) into (73) leads to 


$$
\mathbf{K}_{3}^{+}\left(x_{1}\right)+\mathbf{h}_{3 \times 3}^{-1} \overline{\mathbf{h}}_{3 \times 3} \mathbf{K}_{3}^{-}\left(x_{1}\right)=-\mathbf{h}_{3 \times 3}^{-1} \boldsymbol{\sigma}_{2}^{p}\left(x_{1}\right) .
$$

Finally, inserting (69) into (41) results in

$$
\int_{L_{c}}\left[\mathbf{K}_{3}^{+}\left(x_{1}\right)-\mathbf{K}_{3}^{-}\left(x_{1}\right)\right] d x_{1}=\mathbf{0} .
$$

Thus sub-problem B has been reduced to an equivalent interface crack problem in elastic anisotropic media governed by (75) and (76).

With the following replacements:

$$
\begin{gathered}
\left(\mathbf{h}, \phi_{, 1}, \mathbf{K}\right) \rightarrow\left(\mathbf{h}_{3 \times 3}, \boldsymbol{\sigma}_{2}, \mathbf{K}_{3}\right), \\
\mathbf{k} \rightarrow\left[k_{I I}, k_{I}, k_{I I I}\right]^{T} .
\end{gathered}
$$

Equations (31)-(61) are still valid, from which one can solve for $\mathbf{K}_{3}(z)$ and the stress intensity factors. However, the intensity factor of electric displacement $k_{D}$ has to be determined separately.

To find $k_{D}$, we rewrite (72) as

$$
D_{2}\left(x_{1}\right)=D_{2}^{a}\left(x_{1}\right)-2 \mathbf{W R}\left(x_{1}\right), x_{1} \in L_{b},
$$

where

$$
\mathbf{W}=-\frac{1}{2}\left(\mathbf{h}_{1 \times 3}+\overline{\mathbf{h}}_{1 \times 3}\right) \mathbf{Q} .
$$

We define $k_{D}$ as

$$
k_{D}=-\lim _{r \rightarrow 0} \sqrt{2 \pi r} \mathbf{W}\left\langle\left\langle\left(1+e^{2 \pi \varepsilon_{\alpha}}\right) r^{-i \varepsilon_{\alpha}}\right\rangle\right\rangle \mathbf{R}\left(x_{1}\right), \quad x_{1} \in L_{b},
$$

which can be further reduced to

$$
k_{D}=-\mathbf{W M} \lim _{r \rightarrow 0} \sqrt{2 \pi r} \mathbf{M}^{-1}\left\langle\left\langle\left(1+e^{2 \pi \varepsilon_{\alpha}}\right) r^{-i \varepsilon_{\alpha}}\right\rangle\right\rangle \mathbf{R}\left(x_{1}\right) .
$$

Using (52), we can rewrite (82) as

$$
k_{D}=-\lambda_{D} \mathbf{k}_{\sigma},
$$

where

$$
\lambda_{D}=\mathbf{W M}
$$

Substituting (80) and (33) $)_{1}$ into (84) yields

$$
\lambda_{D}=-\frac{1}{2}\left(\mathbf{h}_{1 \times 3}+\overline{\mathbf{h}}_{1 \times 3}\right) \mathbf{h}_{3 \times 3} .
$$


If $\mathbf{H}$ is real, $\mathbf{h}$ will be real also. Then (85) becomes

$$
\lambda_{D}=-\mathbf{h}_{1 \times 3} \mathbf{h}_{3 \times 3} .
$$

Equation (83) shows that the intensity factor of electric displacement can be directly expressed in term of the stress intensity factor vector.

To determine the electric field within the crack, (72) is rewritten as

$$
D_{2}^{0}\left(x_{1}\right)=D_{2}^{a}\left(x_{1}\right)+\mathbf{c}_{D} \mathbf{h}_{3 \times 3} \mathbf{K}_{3}^{+}\left(x_{1}\right)+\overline{\mathbf{h}}_{d b} \overline{\mathbf{h}}_{3 \times 3} \mathbf{K}_{3}^{-}\left(x_{1}\right),
$$

where

$$
\mathbf{c}_{D}=\mathbf{h}_{1 \times 3} \mathbf{h}_{3 \times 3}^{-1} .
$$

From (77) one has

$$
\overline{\mathbf{h}}_{3 \times 3} \mathbf{K}_{3}^{-}\left(x_{1}\right)=-\mathbf{h}_{3 \times 3} \mathbf{K}_{3}^{+}\left(x_{1}\right)-\boldsymbol{\sigma}_{2}^{a}\left(x_{1}\right), x_{1} \in L_{c} .
$$

Substituting (88) into (87) leads to

$$
D_{2}^{0}\left(x_{1}\right)=D_{2}^{a}\left(x_{1}\right)-\overline{\mathbf{c}}_{D} \overline{\mathbf{h}}_{3 \times 3} \boldsymbol{\sigma}_{2}^{a}\left(x_{1}\right)+\left(\mathbf{c}_{D}-\overline{\mathbf{c}}_{D}\right) \mathbf{h}_{3 \times 3} \mathbf{K}_{3}^{+}\left(x_{1}\right), \quad x_{1} \in L_{c} .
$$

Equation (89) shows that the electric field within the crack may be singular and oscillatory at the crack tip if $c_{D}$ is complex.

For real $\mathbf{H}$, (89) becomes

$$
D_{2}^{0}\left(x_{1}\right)=D_{2}^{a}\left(x_{1}\right)-\mathbf{h}_{1 \times 3} \boldsymbol{\sigma}_{2}^{a}\left(x_{1}\right) .
$$

Equation (90) shows that the electric fields inside the crack are dependent on the stress vector and the electric displacement on the respective crack faces generated by sub-problem A.

\section{Numerical examples}

We numerically examine the effects of dipole rotation on the electric field inside an interfacial crack and the energy release rate at the crack tip. As shown in Fig.1, we assume that material 1 is PZT-4 and material 2 is PZT-5H. The related material properties are listed in Appendix B. A dipole $\mathbf{p}_{0}$ is located at $z_{0}=a+i a$ with a constant moment magnitude of $p_{0}$ but whose direction angle $\varphi$ varies from 0 to $\pi$. The electric field inside an interfacial permeable crack is shown in Figures 3 and 4, 
where $\hat{D}_{2}^{0}\left(x_{1}\right)=10^{10} a^{2} D_{2}^{0}\left(x_{1}\right) / p_{0}$, and the data points are taken within a range $x_{1} / a=0 \ldots 0.99$. In this range, oscillatory behaviour is not visible. The electric field $E_{2}^{0}\left(=D_{2}^{0} / \varepsilon_{0}\right.$ with $\varepsilon_{0}$ being the dielectric constant of air) reaches the maximum value near the right crack tip for the direction of the dipole parallel to the crack, while near $x_{1} / a=0.25$ for the direction of the dipole perpendicular to the crack. The variations of the energy release rate with $\varphi$ for the three crack models are shown in Fig. 5 for $\hat{G}=4 \pi a p_{0}^{2} G \times 10^{10}$. It can be seen that the energy release rates for the three crack models all reach their maximum values when the dipole direction is perpendicular to the crack. Moreover, the energy release rate for a conducting crack is greater than that for an impermeable or permeable crack of the same size under the same loading conditions.

\section{Concluding remarks}

We have analysed the generalized 2D problem of an interface crack between two dissimilar piezoelectric materials with one of the materials subjected to a line dipole. The problem is solved by means of the Stroh formalism. Closed-form solutions for the complex potentials, the intensity factors of fields and the energy release rate are presented, respectively, for impermeable, permeable and conducting cracks. Numerical results are given to show the effect of the dipole rotation on the electric field inside the crack and the energy release rate at the crack tip. These results can be used as fundamental solutions for micromechanics modelling of domain switching in piezoelectric interface fracture.

It should be noted that a spontaneous polarization is associated with a spontaneous strain field and the relationship between them is generally nonlinear. Recently, Wang et al. [17] have conducted 
phase field simulations of the mechanical behavior of a perfect ferroelectric crystal, in which how to calculate the stress field is given in detail. If the strain field associated with an electric dipole is modeled as a force couple, as Fulton and Gao [2] did, the solution for the force couple can be easily written out based on the present results. For example, for an out-plane force couple (vector) in a homogeneous piezoelectric medium, the singular function, similar to (A3), has been given by Gao and Balke [5]

$$
\mathbf{F}_{1 s}(z)=m_{0}\left\langle\left\langle\frac{\varphi_{\alpha}}{\left(z-z_{\alpha 0}\right)^{2}}\right\rangle\right\rangle \mathbf{q}^{\prime},
$$

where $m_{0}$ stands for the magnitude of the force couple, and $\mathbf{q}^{\prime}$ is a known constant vector. Thus, as long as one makes the following replacements:

$$
p_{0} \rightarrow m_{0}, \mathbf{q} \rightarrow \mathbf{q}^{\prime},
$$

the solutions for the interaction of the force couple with an interfacial crack piezoelectric media can easily be obtained from the above results of an electric dipole for the above three crack models. These solutions together with those for the case of an electric dipole can be used to explore the effect of domain switching on interface fracture of piezoelectric materials.

\section{Acknowledgments}

This work was supported by a grant from the Research Grants Council of the Hong Kong Special Administrative Region, China. TY Zhang thanks the Croucher Foundation for the Croucher Senior Research Fellowship Award, which gave him more research time by releasing him from teaching duties. 


\section{Appendix A: Solution to sub-problem A}

For sub-problem A shown in Fig.2, the complex potentials in the upper and lower half-planes have the form

$$
\begin{aligned}
& \mathbf{F}_{1 a}(z)=\mathbf{F}_{1 s}(z)+\mathbf{F}_{10}(z), \quad z \in s_{1}, \\
& \mathbf{F}_{2 a}(z)=\mathbf{F}_{20}(z), \quad z \in S_{2},
\end{aligned}
$$

where $\mathbf{F}_{10}(z)$ and $\mathbf{F}_{20}(z)$ are two holomorphic functions in $s_{1}$ and $s_{2}$, respectively, and $\mathbf{F}_{1 s}(z)$ is the complex potential of an infinite plane subjected to a line dipole at $z_{0}$.

Since the line dipole can be taken as the limiting case of two parallel line charges $e$ of opposite signs at an infinitely small distance $d$ from each other, $\mathbf{F}_{1 s}(z)$ can be obtained by taking the limit of $d \rightarrow 0$ while keeping $e \times d=p_{0}$ as a constant. The solution is [4,5]

$$
\mathbf{F}_{1 s}(z)=p_{0}\left\langle\left\langle\frac{\varphi_{\alpha}}{\left(z-z_{\alpha 0}\right)^{2}}\right\rangle\right\rangle \mathbf{q}, \quad z \in s_{1},
$$

where

$$
\varphi_{\alpha}=\cos \varphi+\mu_{\alpha} \sin \varphi, \quad \mathbf{q}=\frac{1}{2 \pi i} \mathbf{A}_{1}^{T} \mathbf{i}_{4}, i_{4}=(0,0,01)^{T}
$$

Substituting (A1) and (A2) into (16) and (17), and using (13) results in

$$
\begin{gathered}
\mathbf{B}_{1}\left[\mathbf{F}_{10}^{+}\left(x_{1}\right)+\mathbf{F}_{1 s}^{-}\left(x_{1}\right)\right]+\overline{\mathbf{B}}_{1}\left[\overline{\mathbf{F}}_{10}^{-}\left(x_{1}\right)+\overline{\mathbf{F}}_{1 s}^{+}\left(x_{1}\right)\right]=\mathbf{B}_{2} \mathbf{F}_{20}^{-}\left(x_{1}\right)+\overline{\mathbf{B}}_{2} \overline{\mathbf{F}}_{20}^{+}\left(x_{1}\right), \\
\mathbf{Y}_{\mathbf{1}} \mathbf{B}_{1}\left[\mathbf{F}_{10}^{+}\left(x_{1}\right)+\mathbf{F}_{1 s}^{-}\left(x_{1}\right)\right]-\overline{\mathbf{Y}_{\mathbf{1}} \mathbf{B}_{\mathbf{1}}}\left[\overline{\mathbf{F}}_{10}^{-}\left(x_{1}\right)+\overline{\mathbf{F}}_{1 s}^{+}\left(x_{1}\right)\right]=\mathbf{Y}_{2} \mathbf{B}_{2} \mathbf{F}_{20}^{-}\left(x_{1}\right)-\overline{\mathbf{Y}_{2} \mathbf{B}_{2}} \overline{\mathbf{F}}_{20}^{+}\left(x_{1}\right), \\
-\infty<x_{1}<+\infty
\end{gathered}
$$

From (A4) we obtain

$$
\begin{array}{ll}
\mathbf{B}_{1} \mathbf{F}_{10}(z)-\overline{\mathbf{B}}_{2} \overline{\mathbf{F}}_{20}(z)+\overline{\mathbf{B}}_{1} \overline{\mathbf{F}}_{1 s}(z)=\mathbf{0}, & z \in s_{1}, \\
\mathbf{B}_{2} \mathbf{F}_{20}(z)-\overline{\mathbf{B}}_{1} \overline{\mathbf{F}}_{10}(z)-\mathbf{B}_{1} \mathbf{F}_{1 s}(z)=\mathbf{0}, \quad z \in s_{2} .
\end{array}
$$

Similarly, (A5) produces

$$
\mathbf{Y}_{1} \mathbf{B}_{1} \mathbf{F}_{10}(z)+\overline{\mathbf{Y}_{2} \mathbf{B}_{2}} \overline{\mathbf{F}}_{20}(z)-\overline{\mathbf{Y}}_{1} \overline{\mathbf{B}}_{1} \overline{\mathbf{F}}_{1 s}(z)=\mathbf{0}, \quad z \in s_{1},
$$




$$
\mathbf{Y}_{2} \mathbf{B}_{2} \mathbf{F}_{20}(z)+\overline{\mathbf{Y}_{1} \mathbf{B}_{1}} \overline{\mathbf{F}}_{10}(z)-\mathbf{Y}_{1} \mathbf{B}_{1} \mathbf{F}_{1 s}(z)=\mathbf{0}, \quad z \in s_{1} .
$$

Combining (A6) and (A8), we find

$$
\mathbf{B}_{1} \mathbf{F}_{10}(z)=\mathbf{H}^{-1}\left(\mathbf{Y}_{1}-\overline{\mathbf{Y}}_{2}\right) \overline{\mathbf{B}}_{1} \overline{\mathbf{F}}_{1 s}(z), \quad z \in s_{1}
$$

Similarly, (A7) and (A9) give

$$
\mathbf{B}_{2} \mathbf{F}_{20}(z)=\overline{\mathbf{H}}^{-1}\left(\mathbf{Y}_{1}+\overline{\mathbf{Y}}_{1}\right) \mathbf{B}_{1} \mathbf{F}_{1 s}(z), \quad z \in s_{2} .
$$

Substituting (A10) and (A11) into (A1) and (A2) leads to

$$
\begin{gathered}
\mathbf{B}_{1} \mathbf{F}_{1 a}(z)=\mathbf{B}_{1} \mathbf{F}_{1 s}(z)+\mathbf{H}^{-1}\left(\mathbf{Y}_{1}-\overline{\mathbf{Y}}_{2}\right) \overline{\mathbf{B}}_{1} \overline{\mathbf{F}}_{1 s}(z), \quad z \in s_{1}, \\
\mathbf{B}_{2} \mathbf{F}_{2 a}(z)=\overline{\mathbf{H}}^{-1}\left(\mathbf{Y}_{1}+\overline{\mathbf{Y}}_{1}\right) \mathbf{B}_{1} \mathbf{F}_{1 s}(z), \quad z \in s_{2} .
\end{gathered}
$$

Substituting (A12) or (A13) into (14) and (15), one can determine all the field variables in $s_{1}$ and $s_{2}$ for sub-problem B. For example, on the $x_{1}$-axis, the generalized traction vector induced by the dipole can be calculated from (14) using (A13)

$$
\phi_{a, 1}\left(x_{1}\right)=2 \operatorname{Re}\left[\mathbf{N F}_{1 s}\left(x_{1}\right)\right]
$$

where

$$
\mathbf{N}=\overline{\mathbf{H}}^{-1}\left(\mathbf{Y}_{1}+\overline{\mathbf{Y}}_{1}\right) \mathbf{B}_{1}
$$

Inserting (A3) into (A14) we find

$$
\phi_{a, 1}\left(x_{1}\right)=p_{0}\left[\mathbf{N}\left\langle\left\langle\frac{\varphi_{\alpha}}{\left(x_{1}-z_{\alpha 0}\right)^{2}}\right\rangle\right) \mathbf{q}+\overline{\mathbf{N}}\left\langle\left\langle\frac{\bar{\varphi}_{\alpha}}{\left(x_{1}-\bar{z}_{\alpha 0}\right)^{2}}\right\rangle\right) \overline{\mathbf{q}}\right] .
$$

\section{Appendix B: material properties}

The properties of material 1 (PZT-4) are

$$
\begin{array}{lll}
c_{11}=13.90 \times 10^{10} \mathrm{~N} / \mathrm{m}^{2}, & c_{12}=7.78 \times 10^{10} \mathrm{~N} / \mathrm{m}^{2}, & c_{13}=7.74 \times 10^{10} \mathrm{~N} / \mathrm{m}^{2}, \\
c_{33}=11.30 \times 10^{10} \mathrm{~N} / \mathrm{m}^{2}, & c_{44}=2.56 \times 10^{10} \mathrm{~N} / \mathrm{m}^{2}, & \\
e_{31}=-6.98 \mathrm{C} / \mathrm{m}^{2}, & e_{33}=13.84 \mathrm{C} / \mathrm{m}^{2}, & e_{15}=13.44 \mathrm{C} / \mathrm{m}^{2}, \\
\varepsilon_{11}=60.00 \times 10^{-10} \mathrm{C} / \mathrm{Vm}, & \varepsilon_{33}=54.70 \times 10^{-10} \mathrm{C} / \mathrm{Vm} . &
\end{array}
$$


The properties of material 2 (PZT-5H) are

$$
\begin{array}{lll}
c_{11}=12.60 \times 10^{10} \mathrm{~N} / \mathrm{m}^{2}, & c_{12}=5.50 \times 10^{10} \mathrm{~N} / \mathrm{m}^{2}, & c_{13}=5.30 \times 10^{10} \mathrm{~N} / \mathrm{m}^{2}, \\
c_{33}=11.70 \times 10^{10} \mathrm{~N} / \mathrm{m}^{2}, & c_{44}=3.53 \times 10^{10} \mathrm{~N} / \mathrm{m}^{2}, & \\
e_{31}=-6.50 \mathrm{C} / \mathrm{m}^{2}, & e_{33}=23.30 \mathrm{C} / \mathrm{m}^{2}, & e_{15}=17.00 \mathrm{C} / \mathrm{m}^{2}, \\
\varepsilon_{11}=151.00 \times 10^{-10} \mathrm{C} / \mathrm{Vm}, & \varepsilon_{33}=130.00 \times 10^{-10} \mathrm{C} / \mathrm{Vm} . &
\end{array}
$$

\section{References}

[1] Zhang, TY, Zhao, MH, Tong, P. Fracture of Piezoelectric ceramics. Adv Appl Mech 2000; 38: $147-289$.

[2] Fulton, CC, Gao, H. Microstructural modelling of ferroelectric fracture. Acta mater 2001; 49: 2039-2054.

[3] Fu, R, Zhang, TY. Influence of temperature and electric field on the bending strength of lead zirconate titanate ceramics. Acta Mater 2000; 48: 1729-1740.

[4] Wang, JW, Kuang, ZB. The interaction between crack and electric dipole of Piezoelectricity. Acta Mech Solida Sin 2000; 13: 283-289.

[5] Gao, CF, Balke, H. An electric dipole and a force couple near a permeable crack in a piezoelectric material. Acta Mech 2003; 162: 57-67.

[6] Ting, TCT. Anisotropic Elasticity: Theory and Applications. Oxford Science Publications, New York, 1996.

[7] Zhang, TY, Gao, CF. Fracture behaviors of piezoelectric materials. Theor Appl Fract Mech 2004; 41: 339-379. 
[8] Pak, YE. Crack extension force in a piezoelectric material. ASME J Appl Mech 1990; 57: 647653.

[9] Muskhelishvili, NI. Some basic problems of mathematical theory of elasticity. Noordhoof, leyden, 1975.

[10] $\mathrm{Wu}, \mathrm{KC}$. Stress intensity factors and energy release rate for interfacial cracks between dissimilar anisotropic materials. ASME J Appl Mech 1990; 57: 882-886.

[11] Zhang, TY, Lee, SB. Stress intensity factors of interfacial cracks. Eng Fract Mech 1993; 44: 539-544.

[12] Cherepanov, GP (1974). Mechanics of brittle fracture. Nauka. Moscow (in Russian). English translation: McGraw-Hill, 1979.

[13] Yeh, CS, Shu, YC, Wu, KC. Conservation laws in anisotropic elasticity I. Basic framework. Proc R Soc Lond 1993; A443: 139-151.

[14] Beom, HG, Atluri, SN. Near-tip fields and intensity factors for interfacial cracks in dissimilar anisotropic piezoelectric media. Int J Fract 1996; 75: 163-183.

[15] Homulka, TA, Keer, LM. A Mathematical Solution of a Special Mixed Boundary Value Problem of Anisotropic Elasticity. Q J Mech Appl Math 1995; 48:635-658.

[16] Parton, VZ, Kudryavtsev, BA. Electromagnetoelasticity. New York, Gordon and Breacn, 1998.

[17] Wang, J, Shi, SQ, Chen, LQ, Li, YL, Zhang, TY. Phase field simulations of ferroelectric/ferroelastic polarization switching. Acta Mater 2004; 52: 749-764. 


\section{List of Figure Captions}

Fig.1 A dipole interacting with an interfacial crack

Fig.2. A dipole in a bi-material system

Fig.3. Electric field inside the crack for $\varphi=0$ and $\pi$

Fig.4. Electric field inside the crack for $\varphi=\pi / 3$ and $\pi / 2$

Fig.5. Energy release rate for three crack models 


\section{$\underline{\text { List of Figures }}$}

Fig.1. A dipole interacting with an interfacial crack

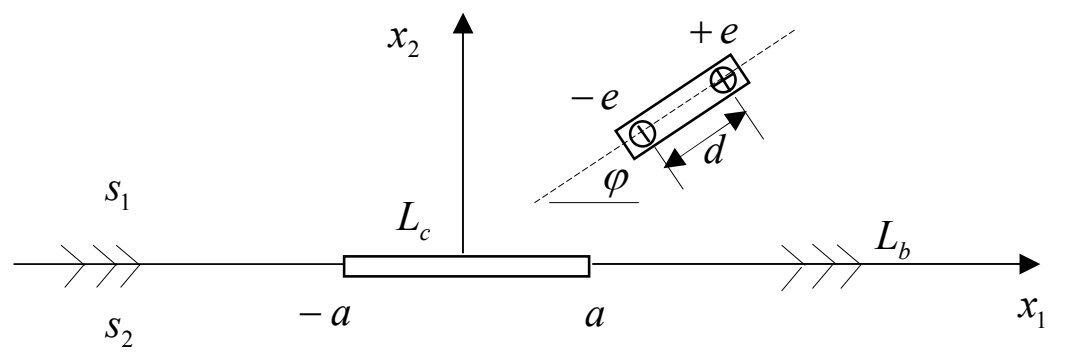


Fig.2. A dipole in a bi-material system

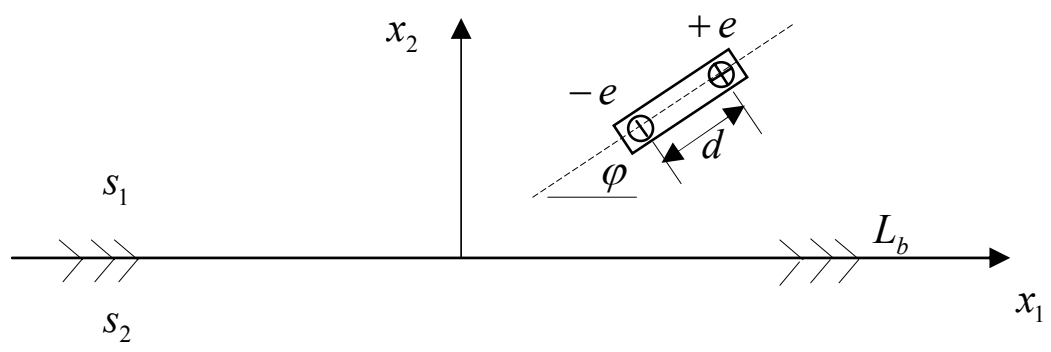


Fig.3. Electric field inside the crack for $\varphi=0$ and $\pi$

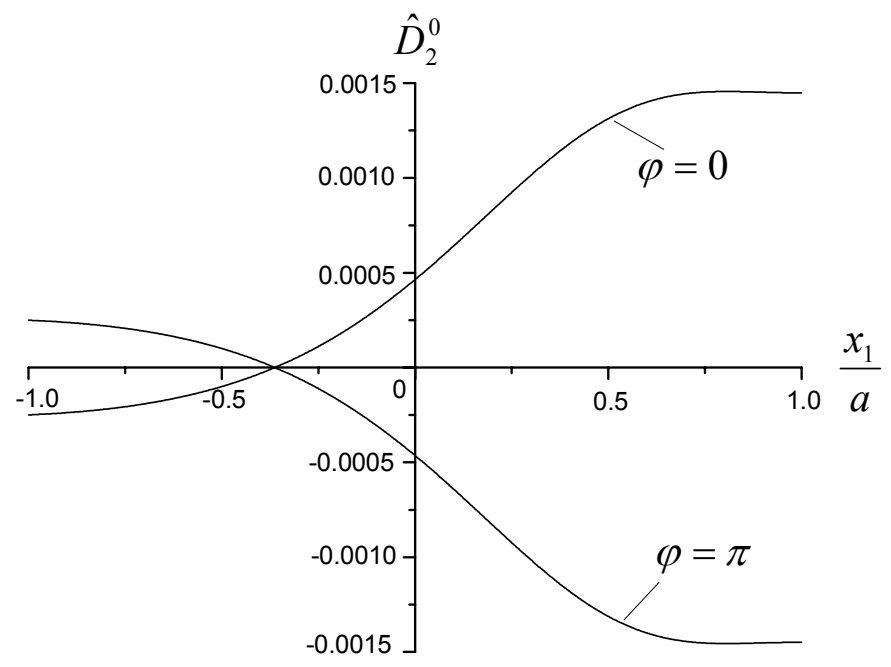


Fig.4. Electric field inside the crack for $\varphi=\pi / 3$ and $\pi / 2$

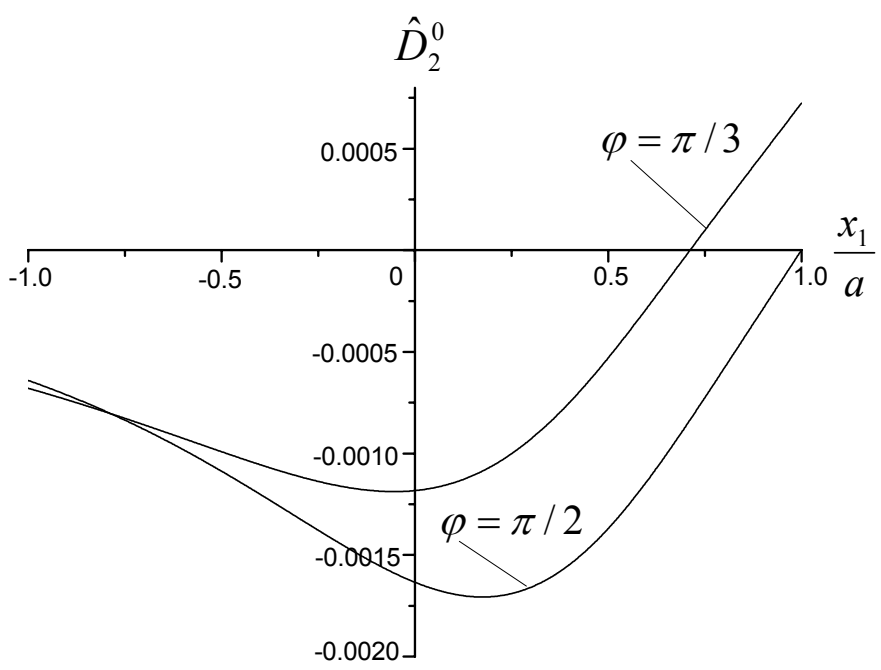


Fig.5. Energy release rate for three crack models

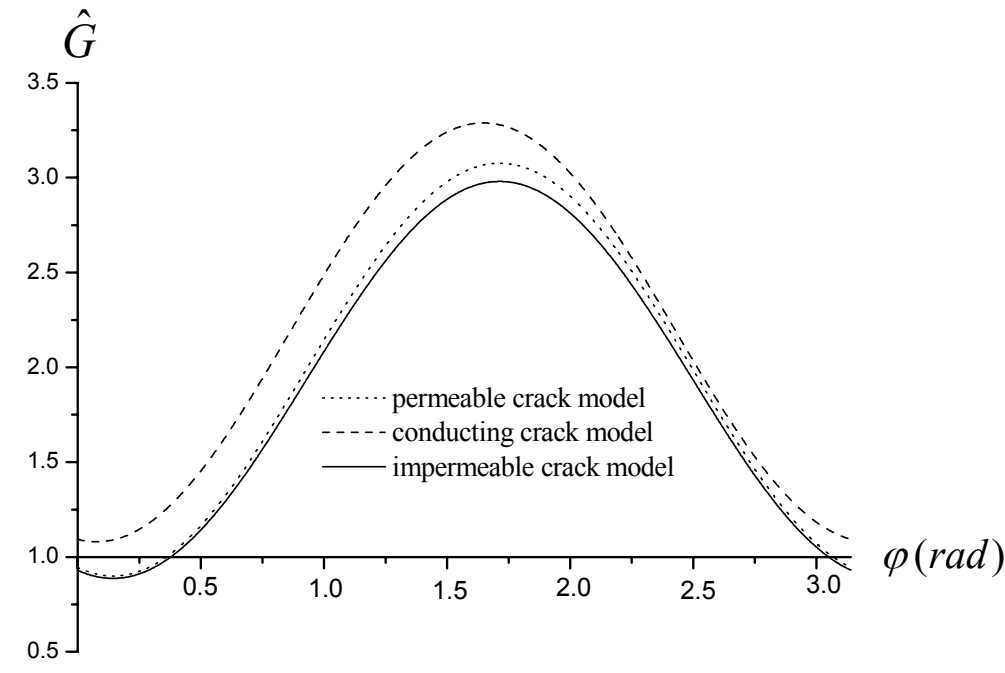

